\title{
Size-Dependent Joule Heating of Gold Nanoparticles Using Capacitively Coupled Radiofrequency Fields
}

\author{
Christine H. Moran ${ }^{1,2, \uparrow}$, Sean M. Wainerdi ${ }^{2,3, \uparrow}$, Tonya K. Cherukuri ${ }^{4}$, Carter Kittrell ${ }^{4}$, Benjamin J. Wiley ${ }^{5}$, \\ Nolan W. Nicholas ${ }^{6}$, Steven A. Curley ${ }^{2,7}$, John S. Kanzius, and Paul Cherukuri, ${ }^{2,4,5,8}(\varangle)$ \\ ${ }^{1}$ Department of Bioengineering, Rice University, 6100 Main Street, Houston, TX 7005, USA \\ ${ }^{2}$ Department of Surgical Oncology, MD Anderson Cancer Center, 1515 Holcombe Blvd, Box 107, Houston, TX 77030, USA \\ ${ }^{3}$ Department of Bioengineering, Texas A\&M University, College Station, TX 77843, USA \\ ${ }^{4}$ Department of Chemistry and Richard E. Smalley Institute for Nanoscale Science and Technology, Rice University, 6100 Main Street, \\ Houston, TX 77005, USA \\ ${ }^{5}$ Department of Chemistry and Chemical Biology, Harvard University, 12 Oxford Street, Cambridge, MA 02138, USA \\ ${ }^{6}$ Department of Physics and Richard E. Smalley Institute for Nanoscale Science and Technology, Rice University, 6100 Main Street, \\ Houston, TX 77005, USA \\ ${ }^{7}$ Department of Mechanical Engineering and Material Science, Rice University, 6100 Main Street, Houston, TX 77005, USA \\ ${ }^{8}$ Department of Experimental Therapeutics, MD Anderson Cancer Center, 1515 Holcombe Blvd, Box 107, Houston, TX77030, USA \\ Received: 5 February 2009 / Revised: 4 April 2009 / Accepted: 6 April 2009 \\ (C) Tsinghua University Press and Springer-Verlag 2009. This article is published with open access at Springerlink.com
}

\begin{abstract}
Capacitively coupled shortwave radiofrequency fields $(13.56 \mathrm{MHz})$ resistively heat low concentrations $(\sim 1$ ppm) of gold nanoparticles with a thermal power dissipation of $\sim 380 \mathrm{~kW} / \mathrm{g}$ of gold. Smaller diameter gold nanoparticles $(<50 \mathrm{~nm})$ heat at nearly twice the rate of larger diameter gold nanoparticles $(\geqslant 50 \mathrm{~nm})$, which is attributed to the higher resistivity of smaller gold nanostructures. A Joule heating model has been developed to explain this phenomenon and provides critical insights into the rational design and engineering of nanoscale materials for noninvasive thermal therapy of cancer.
\end{abstract}

\section{KEYWORDS}

Resistivity, radiofrequency, gold, nanoparticles, cancer, thermal

\section{Introduction}

Nanoparticle-enhanced thermal destruction of tumors has been demonstrated by using near infrared (NIR) light to heat gold nanoshells or by using strong alternating magnetic fields to heat iron oxide nanoparticles [1-3]. Although these methods show significant promise in treating cancer, they have disadvantages that limit their practical implementation as therapies. Noninvasive NIR heating of nanoshells is only effective at treating subsurface cancer ( a few mm deep) due to significant scattering and attenuation of NIR light by biological tissues [4]. Magnetic field-based hyperthermia

Address correspondence to pcherukuri@mdanderson.org

${ }^{\dagger}$ These two authors made an equal contribution to the work. 
is effective at treating deep tissue cancer, but suffers from limited thermalization by iron oxide nanoparticles. The highest reported thermal power dissipation by iron oxide is a relatively low $\sim 500 \mathrm{~W} / \mathrm{g}$ of nanomaterial (at a magnetic field amplitude of $\sim 11$ $\mathrm{kA} / \mathrm{m}$ ). Therefore, the concentrations of iron oxide required for effective therapy are much higher than can be reasonably achieved in vivo $[3,5]$.

Recently, we have shown that gold nanoparticles heat under capacitively coupled radio frequency fields $[6,7]$. RF heating of gold nanoparticles within cancer cells overcomes the major limitations associated with other noninvasive nanoparticle heating methods, since RF energy penetrates well into the body to efficiently heat gold nanoparticles within deep tissue tumors [8]. Previous studies evaluated the therapeutic benefits of capacitive RF heating of gold nanoparticles, but the mechanism of thermalization of RF energy by gold nanoparticles has remained unaddressed and poorly understood.

This paper aims to clarify the physical basis of gold nanoparticle heating by examining the capacitive RF heating properties of gold nanoparticles with respect to their volume fraction and diameter. The analysis contained herein provides critical insights into how the physical properties of gold nanoparticles influence their RF thermal delivery, which will aid in the further development of nanoscale materials for the treatment of cancer.

\section{Experimental}

\subsection{Materials}

Aqueous spherical gold nanoparticles with diameters between 5 and $250 \mathrm{~nm}$ were purchased from Ted Pella, Inc. (Redding, CA, USA). Gold nanoshells, provided by Nanospectra Biosciences, Inc. (Houston, TX, USA), consist of a $120 \mathrm{~nm}$ diameter silica core (Precision Colloids, LLC., Cartersville, GA, USA) with a 10-15 nm thick outer shell of gold $(\sim 10-15 \mathrm{~nm}$ thick).

\subsection{Materials characterization}

The size and morphology of all nanoparticles were confirmed using transmission electron microscopy
(TEM). TEM was performed using a JEM 1010 transmission electron microscope (JEOL, Inc., Peabody, MA, USA) with an accelerating voltage of $80 \mathrm{kV}$ and digital images were acquired using an AMT Imaging System (Advanced Microscopy Techniques Corp., Danvers, MA, USA). Drops of nanoparticle suspensions were placed onto a poly$L$-lysine treated formvar coated copper grid for $1 \mathrm{~h}$. Grids were blotted dry with filter paper and air dried before TEM observation (see Fig. S-1 in the Electronic Supplementray Material (ESM)). All gold concentrations are reported in ppm by mass and were determined via inductively coupled plasmamass spectrometry (ICP-MS) analysis.

\subsection{Capacitively coupled RF heating of nanoparticle solutions}

Figure 1 shows the $13.56 \mathrm{MHz}$ capacitive RF heating system (Therm Med, LLC, Inc., Erie, PA, USA). A cylindrical quartz cuvette $(1 \mathrm{~cm}$ diameter, $1.5 \mathrm{~cm}$ height) containing $1.5 \mathrm{~mL}$ of aqueous colloid was positioned on a thin $(\sim 0.5 \mathrm{~cm})$ horizontal Teflon ${ }^{\circledR}$ platform (not shown) located mid-plane between two insulated copper electrodes at a spacing of 7.5

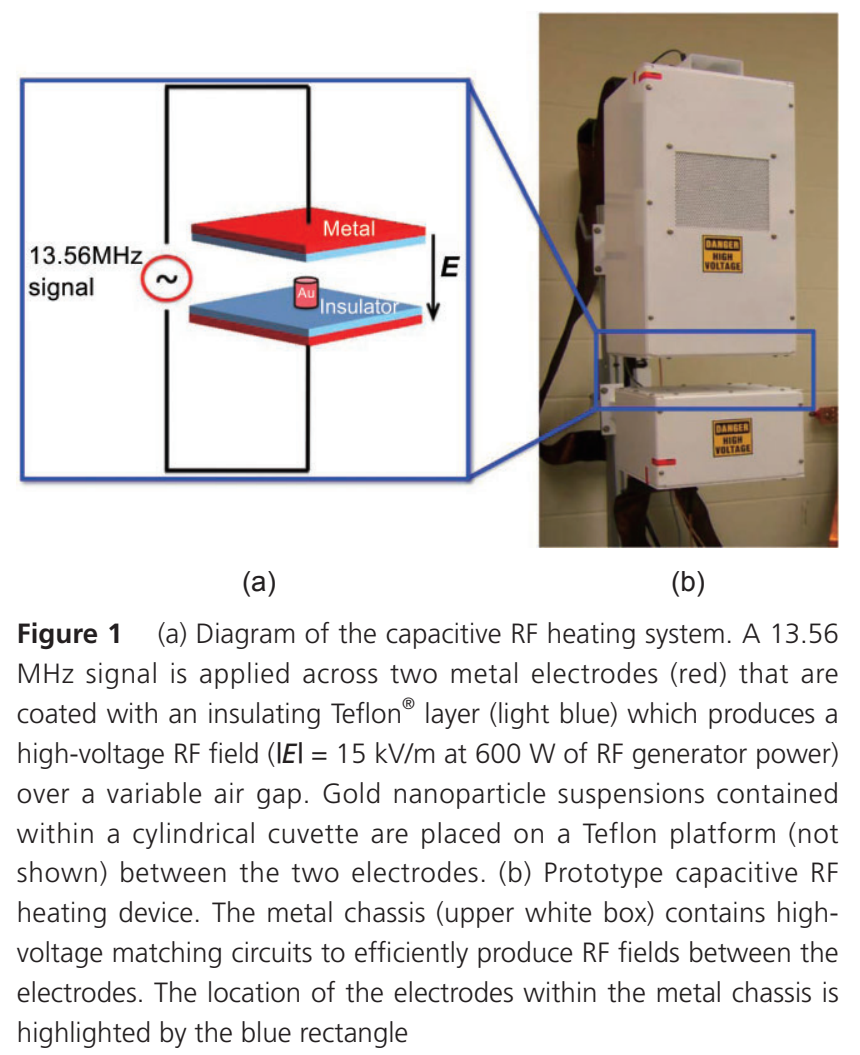

Springer 
$\mathrm{cm}$. All suspensions were exposed to $600 \mathrm{~W}$ of RF generator power resulting in an RF field of $15 \mathrm{kV} / \mathrm{m}$. Reflected RF power was monitored and minimized by impedance matching during RF exposure [7-9].

\subsection{Thermal imaging}

The temperature of the cuvette was continuously monitored using an infrared camera and thermal emissions were recorded (Amber Engineering Inc., Goletta, California, USA). All colloidal suspensions were exposed to RF fields until they reached a final temperature of $70{ }^{\circ} \mathrm{C}$ or for a maximum duration of $2 \mathrm{~min}$, whichever was achieved first.

\section{Results and discussion}

Figure 2 shows a time-lapsed sequence of captured infrared images of an aqueous suspension of 10 $\mathrm{nm}$ gold nanoparticles (36 ppm) capacitively coupled to an RF field. Upon RF absorption, the temperature of the gold nanoparticle suspension rapidly increased from 25 to $70^{\circ} \mathrm{C}$ in less than $20 \mathrm{~s}$ and the solution boiled within $30 \mathrm{~s}$ of RF exposure. The false color thermal map shows uniform heating of the suspension with a sharp temperature gradient at the base of the cuvette. The thin non-conducting platform supporting the cuvette remained at room

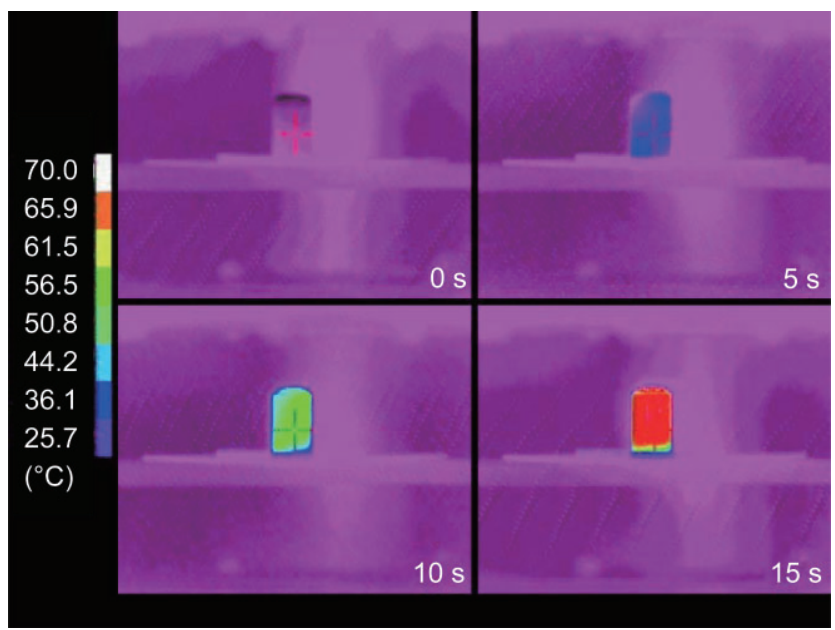

Figure 2 Time-lapsed infrared images, taken $5 \mathrm{~s}$ apart, of a cylindrical quartz cuvette filled with $1.5 \mathrm{~mL}$ of $10 \mathrm{~nm}$ gold nanoparticles in water ( $36 \mathrm{ppm}$ ) exposed to a $15 \mathrm{kV} / \mathrm{m}$ RF field. RF energy is absorbed by gold, which boils water in less than $30 \mathrm{~s}$. The temperature of each sample was recorded at the center of the crosshair temperature throughout RF exposure.

Figure 3 plots the change in temperature versus time for selected concentrations of $10 \mathrm{~nm}$ gold nanoparticles capacitively coupled to RF fields. Deionized water alone and physiological saline (not shown) exhibited a $5{ }^{\circ} \mathrm{C}$ rise from room temperature over a 2-min period, which equates to $0.2 \mathrm{~W}$ of thermal power dissipation. But the addition of just $\sim 1$ ppm of gold by mass, resulted in a five-fold increase in thermal power dissipation, giving a temperature rise $(\Delta T)$ of $35^{\circ} \mathrm{C}$ in $2 \mathrm{~min}$.

The thermal power dissipated per gram of material is the ratio of the thermal energy delivered into solution to the total mass of material in suspension. Therefore, the thermal power dissipation of 1 ppm gold nanoparticles under our RF field conditions was a remarkable $\sim 380 \mathrm{~kW} / \mathrm{g}$. Additionally, for the maximum concentration (36 ppm) of $10 \mathrm{~nm}$ gold nanoparticles, the heating rate was $3.4^{\circ} \mathrm{C} / \mathrm{s}$, which corresponds to a 70 -fold increase in the thermal power dissipation by gold $(14 \mathrm{~W})$ as compared to water alone.

In order to investigate the dependence of RF heating on nanoparticle diameter, we capacitively applied RF fields to various sizes of gold nanoparticles (5-250 nm). Figure 4 displays the heating rates of several different diameters of gold

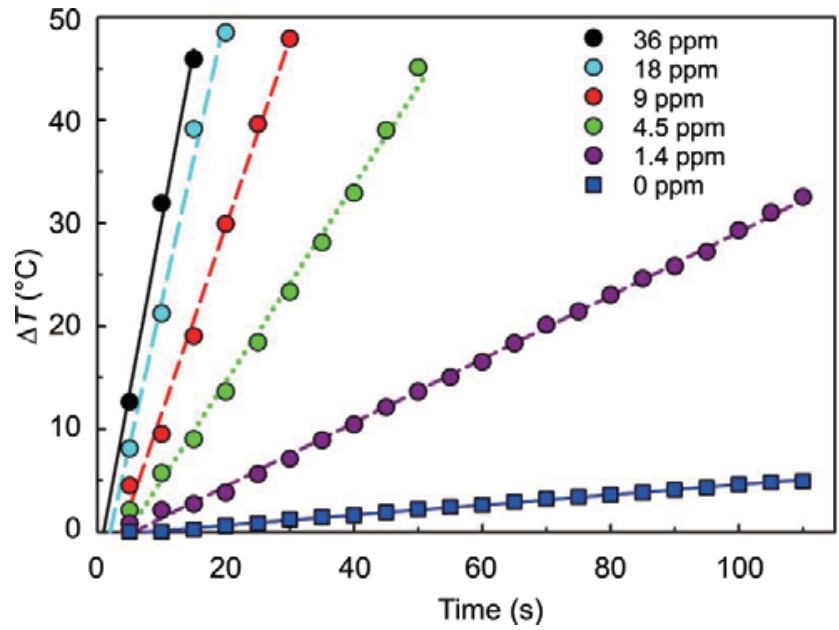

Figure 3 Change in temperature with time of $10 \mathrm{~nm}$ diameter gold nanoparticle suspensions (circles) and water alone (squares) exposed to RF fields. Lines shown are linear fits by least squares regression of temperatures recorded from the infrared images shown in Fig. 2. RF energy absorbed by $1.4 \mathrm{ppm}$ of gold nanoparticles exhibited a final $\Delta T$ $\sim 35^{\circ} \mathrm{C}$ in $2 \mathrm{~min}$, whereas water alone exhibited a $\Delta T \sim 5^{\circ} \mathrm{C}$ 
nanoparticles versus their gold volume fraction. This plot reveals three important properties of gold nanoparticles under capacitively coupled RF fields. First, the rate of RF heating increases nonlinearly and approaches a horizontal asymptote as the nanoparticle concentration increases. This finding is similar to what we observed for the concentrationdependent RF heating of carbon nanotubes [8]. Second, at equivalent volume fractions, small diameter gold nanoparticles $(<50 \mathrm{~nm})$ exhibit heating rates that are approximately twice as high as those of gold nanoparticles with diameters of $50 \mathrm{~nm}$ and above. Third, solid gold nanoparticles with diameters between 50-250 $\mathrm{nm}$ heat at nearly equivalent rates with no distinguishable diameter dependence.

In order to independently examine nanoparticle diameter and gold content effects under RF fields, we tested gold nanoshells. Since nanoshells are composed of an inner dielectric core $\left(\mathrm{SiO}_{2}\right)$ and a thin outer shell of gold, the total volume fraction of gold in solution can be varied while holding the overall diameter of the nanoparticle constant. Surprisingly, at equivalent gold volume fractions, $150 \mathrm{~nm}$ diameter gold nanoshells (ca. 10-15 nm gold shell thickness) exhibit RF heating rates comparable to solid gold nanoparticles $\leqslant 20 \mathrm{~nm}$ in diameter. Note, that in a

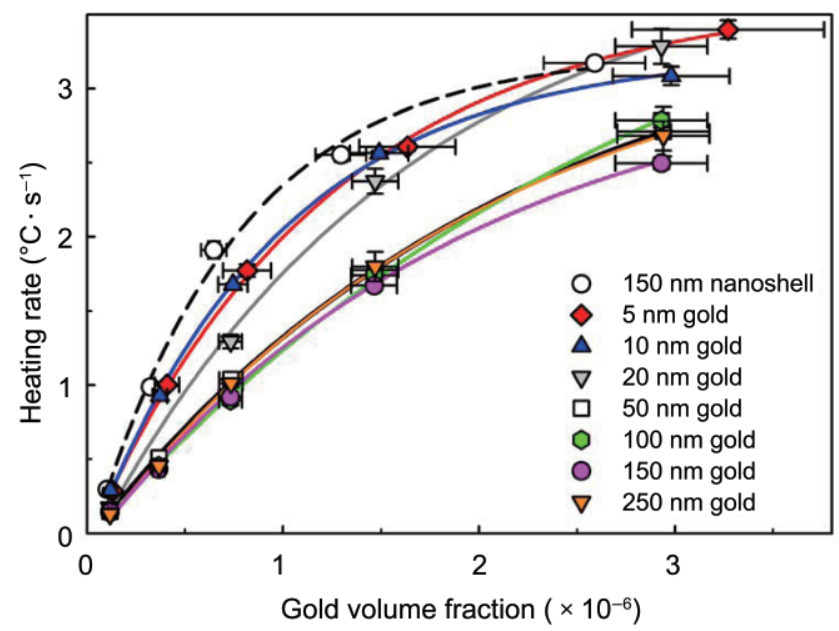

Figure 4 Size-dependent RF heating rates of gold nanoparticles versus gold volume fraction. Heating rates were obtained from the slopes of the linear fits shown in Fig. 3. There is a clear sizedependent clustering of RF heating rates with $150 \mathrm{~nm}$ diameter gold nanoshells (with a shell thickness of 10-15 nm) exhibiting heating rates comparable to smaller solid gold nanoparticles, due to the increased resistivity of smaller gold nanostructures. Note, some data points are obscured by overlapping symbols control experiment $120 \mathrm{~nm}$ diameter uncoated silica nanoparticles exhibited an RF heating rate equivalent to that of water.

The increased RF induced heating rates of gold nanoparticles $<50 \mathrm{~nm}$ in diameter and gold nanoshells 10-15 $\mathrm{nm}$ thick can be explained by the higher resistivity of small metal nanostructures compared to bulk metals [10]. Recent studies have shown that the resistivity of silver nanowires $15 \mathrm{~nm}$ in diameter is approximately twice that of bulk silver. Particles and shells of gold with dimensions on the order of $10 \mathrm{~nm}$ are expected to exhibit a similar increase in resistivity due to increased electron-surface scattering since the size of the metal is significantly smaller than the mean free path of electrons in gold, which is on the order of $50 \mathrm{~nm}$ [10-12].

If the heat released is due to resistive $(\Omega)$ dissipation by gold, then the measured heat should scale with Joule's law, $P=I^{2} R$, where $P$ is the power dissipated as heat, $I$ is the current, and $R$ is the resistance. For a given volume fraction of gold nanoparticles, an increase in the resistivity should lead to a commensurate increase in the amount of heat generated by ohmic dissipation. As such, the observed doubling of the heating rate for small gold nanoparticles and nanoshells is consistent with the hypothesis that gold nanoparticles are heating resistively under capacitively coupled RF fields.

Radiofrequencies are far below the electronic resonance frequencies for gold nanoparticles, which are typically in the infrared-visible spectrum; because of this, capacitive coupling to the RF field dominates the movement of charge through nanoparticles. Therefore, the amount of Joule heat generated by each gold nanoparticle under RF oscillations can be directly estimated by approximating gold nanoparticles as conductors of constant cross section:

$$
I^{2} R=\sigma(\omega) \cdot\left[a \cdot d \cdot E^{2}\right]
$$

where $\sigma(\omega)$ is the Drude model conductivity of gold $\left(\sim 4.3 \times 10^{7} \Omega^{-1} \cdot \mathrm{m}^{-1}\right.$ at an angular frequency of $85.16 \mathrm{MHz}), E$ is the magnitude of the electric field, $a$ is the cross-sectional area of the gold nanoparticle, and $d$ is its diameter [13-15]. Assuming efficient heat transfer from $n$ nanoparticles to the surrounding 
water, the volumetric RF induced heating rate $(\mathrm{d} T /$ $\mathrm{d} t$ ) of gold nanoparticles is shown in Eq. (2):

$$
\frac{\mathrm{d} T}{\mathrm{~d} t}=\left[\frac{\sigma(\omega) \cdot a \cdot d \cdot|\boldsymbol{E}|^{2}}{v \cdot c_{\mathrm{w}}}\right] \cdot n
$$

where $v$ is the volume of the suspension and $c_{\mathrm{w}}$ is the heat capacity of water $\left(4.18 \mathrm{~J} \cdot \mathrm{mL}^{-1} \cdot{ }^{\circ} \mathrm{C}^{-1}\right)[16]$.

A $1.5 \mathrm{~mL}$ suspension of $100 \mathrm{~nm}$ diameter gold nanoparticles $\left(n=9 \times 10^{9}\right)$ exposed to an effective RF field strength in water of $|E| \sim 200 \mathrm{~V} / \mathrm{m}$ exhibited an experimental heating rate of $\sim 2.8^{\circ} \mathrm{C} / \mathrm{s}$, whilst Eq. (2) yields a comparable RF induced theoretical heating rate of $1.9{ }^{\circ} \mathrm{C} / \mathrm{s}$. The agreement between the theoretical and experimental RF induced heating rates, as well as the increased heating observed for smaller gold nanostructures, provides further evidence that Joule heating of gold nanoparticles by capacitively coupled RF fields is involved.

\section{Conclusions}

In summary, gold nanoparticles at low concentrations deliver a remarkable $\sim 380 \mathrm{~kW} / \mathrm{g}$ of thermal power dissipation under capacitively coupled RF fields (13.56 MHz). Gold nanoparticles resistively heat under RF fields, with small gold nanoparticles $(\leqslant 20$ $\mathrm{nm}$ ) and thin gold nanoshells (shell thickness of $\sim 10-$ $15 \mathrm{~nm}$ ) exhibiting heating rates approximately twice that of gold nanoparticles of $50 \mathrm{~nm}$ or larger, due to the higher resistivity of smaller nanostructures. By quantifying the amount of heat produced by gold nanoparticles as a function of size and concentration, this study establishes the critical design metrics necessary for formulating RF responsive nanoscale materials to enhance the noninvasive thermal destruction of cancer.

\section{Acknowledgements}

This work was supported by the Radiowave Therapy Fund and Institutional Core Grant \#CA16672 HighResolution Electron Microscopy Facility, UTMDACC. The authors are grateful to Richard E. Smalley, Boris Yakobson, Bruce Johnson, Robert Curl, and George M. Whitesides for helpful discussions and suggestions. The authors are also grateful to Nanospectra
Biosciences, Inc., Donald Payne, and Glenn Goodrich for providing gold nanoshell samples and Precision Colloids, LLC for providing silica samples.

Electronic Supplementary Material: Supplementary material (one figure showing transmission electron micrographs of $10 \mathrm{~nm}$ diameter solid gold nanoparticles and $150 \mathrm{~nm}$ diameter gold nanoshells) is available in the online version of this article at http://dx.doi.org/10.1007/s12274-009-9048-1 and is accessible free of charge.

\section{References}

[1] Hirsch, L. R.; Stafford, R. J.; Bankson, J. A.; Sershen, S. R.; Rivera, B.; Price, R. E.; Hazle, J. D.; Halas, N. J.; West, J. L. Nanoshell-mediated near infrared thermal therapy of tumors under magnetic resonance guidance. P. Natl. Aacd. Sci. USA 2003, 100, 13549-13554.

[2] Gobin, A. M.; Lee, M. H.; Halas, N. J.; James, W. D.; Drezek, R. A.; West, J. L. Near infrared resonant nanoshells for combined optical imaging and photothermal cancer therapy. Nano Lett. 2007, 7, 1929 1934.

[3] Hergt, R.; Hiergeist, R.; Hilger, I.; Kaiser, W. A.; Lapatnikov, Y.; Margel, S.; Richter, U. Maghemite nanoparticles with very high AC-losses for application in RF-magnetic hyperthermia. J. Magn. Magn. Mater. 2004, 270, 345-357.

[4] Arnfield, M. R.; Mathew, R. P.; Tulip, J.; McPhee, M. S. Analysis of tissue optical coefficients using an approximate equation valid for comparable absorption and scattering. Phys. Med. Biol. 1992, 37, 1219-1230.

[5] Kalambur, V. S.; Longmire, E. K.; Bischof, J. C. Cellular level loading and heating of superparamagnetic iron oxide nanoparticles. Langmuir 2005, 23, 12329-12336.

[6] Curley, S. A.; Cherukuri, P.; Briggs, K.; Patra, C. R.; Upton, M.; Dolson, E.; Mukherjee, P. Noninvasive radiofrequency field induced hyperthermic cytotoxicity in human cancer cells using cetuximab-targeted gold nanoparticles. J. Exp. Ther. Oncol. 2008, 7, 313-326.

[7] Gannon, C. J.; Patra, C. R; Bhattacharya, R., Mukerjee, P.; Curley, S. A. Intracellular gold nanoparticles enhance non-invasive radiofrequency thermal destruction of human gastrointestinal cancer cells. J. Nanobiotechnol. 2008, 6, 2. 
[8] Gannon, C. J.; Cherukuri, P.; Yakobson, B. I.; Cognet, L.; Kanzius, J. S.; Kittrell, C.; Weisman, R. B.; Pasquali, M.; Schmidt, H. K.; Smalley, R. E.; Curley, S. A. Carbon nanotube-enhanced thermal destruction of cancer cells in a noninvasive radiofrequency field. Cancer 2007, 110, 2654-2665.

[9] Kanzius, J. S. U.S. Patent Pub. Nos. US 2006/0190063 A1, US2005/02511233 A1, US2005/0251234 A1, and World Intellectual Property Organization WO 2007/027614

[10] Wiley, B. J.; Wang, Z.; Wei, J.; Yin, Y.; Cobden, D. H.; $\mathrm{Xia}, \mathrm{Y}$. Synthesis and electrical characterization of silver nanobeams. Nano Lett. 2006, 6, 2273-2278.

[11] Link, S.; El-Sayed, M. A. Spectral properties and relaxation dynamics of surface plasmon electronic oscillations in gold and silver nanodots and nanorods. J.
Phys. Chem. B 1999, 103, 8410-8426.

[12] Link, S.; Burda, C.; Wang, Z. L; El-Sayed, M. A. Electron dynamics in gold and gold-silver nanoparticles: The influence of a non-equilibrium electron distribution and the size dependence of the electron-phonon relaxation. J. Chem. Phys. 1999, 111, 1255-1264.

[13] Kreibig, U. Electronic properties of small silver particles: The optical constants and their temperature dependence. J. Phys. F: Met. Phys. 1974, 4, 999-1014.

[14] Kittel, C. Introduction to Solid State Physics; J. Wiley \& Sons: New York, NY, 2005.

[15] Johnson, P. B.; Christy, R. W. Optical constants of the noble metals. Phys. Rev. B. 1972, 6, 4370-4379.

[16] Metaxas, A. C. Foundations of Electroheat: A Unified Approach; J. Wiley \& Sons: New York, NY, 1996. 